\title{
On decomposition problems on manifolds with a special differential operators
}

\author{
Marek Jukl and Lenka Juklová
}




\title{
ON DECOMPOSITION PROBLEMS ON MANIFOLDS WITH A SPECIAL DIFFERENTIAL OPERATOR
}

\author{
MAREK JUKL AND LENKA JUKLOVÁ
}

\begin{abstract}
This paper deals with the properties of a special differential operator with respect to the general decomposition of tensor fields on manifolds with affine connection. It is shown that properties of a special differential operator are transferred to the components of a given decomposition.
\end{abstract}

2000 Mathematics Subject Classification: Primary 53A55, 15A69; Secondary 53B05.

Keywords: decomposition of tensors, recurrency, differential operator, covariant derivative

\section{INTRODUCTION}

The trace decomposition of tensors on an $n$-dimensional Riemannian manifold $M_{n}$ was described by Weyl [8]. This decomposition problem may be naturally generalized for certain cases (see for example [2,4-7,9]).

In this paper, we generalize some results from [3] concerning decompositions of tensors and tensor fields. Crasmareanu [1] has studied decompositions of tensor fields for the case of covariant derivative of such tensors which satisfy certain conditions. We consider a special differential operator and generalize those results.

\section{GeNerAL DECOMPOSITION OF TENSORS}

It is well known that on every manifold $M_{n}$, a positive definite metric $g$ determining the structure of a Riemannian manifold may be introduced. For practical reasons (e.g., in theoretical physics), the pseudo-Riemannian manifolds with an indefinite pseudometric $g$ are considered, too. In what follows, we will use in both cases the terms of Riemannian manifold and metric.

Let $M_{n}$ be a manifold with a metric $g$. We introduce the following notation: For every ordered system of indices $i_{1}, i_{2}, \ldots, i_{q}, 1 \leq i_{\rho} \leq n$, and every pair $\left(i_{\rho}, i_{\sigma}\right)$, $\rho<\sigma$, of them, we denote by

$$
\stackrel{(\rho, \sigma)}{M} \underset{i_{1}, \cdots i_{\rho-1} \curlyvee i_{\rho+1} \cdots i_{\sigma-1} \curlyvee i_{\sigma+1} \cdots i_{q}}{\stackrel{\rho}{Y}}
$$

The paper was supported by Grant P201/11/0356 of the Czech Science Foundation. 
the tensor of the type $(0, q-2)$, where the $\rho$-th and the $\sigma$-th indices are omitted.

Then we have the following theorem:

Theorem 1. Let $T_{i_{1} \cdots i_{q}}$ be a $(0, q)$-tensor and

$$
\left(i_{k_{1}}, i_{l_{1}}\right),\left(i_{k_{2}}, i_{l_{2}}\right), \ldots,\left(i_{k_{s}}, i_{l_{s}}\right), s \leq \frac{1}{2} q(q-1),
$$

be pairs of indices satisfying $k_{\sigma}<l_{\sigma}$. Then there exists the decomposition of the tensor $T_{i_{1} \cdots i_{q}}$ of the form

$$
T_{i_{1} \cdots i_{q}}=\tilde{T}_{i_{1} \cdots i_{q}}+\sum_{\sigma=1}^{s} g_{i_{k_{\sigma}} i_{l_{\sigma}}} \cdot \stackrel{\left(k_{\sigma}, l_{\sigma}\right)}{M} \underset{i_{1}, \cdots i_{k_{\sigma}-1} \curlyvee k_{i_{\sigma}+1} \cdots i_{l_{\sigma}-1} \curlyvee i_{l_{\sigma}+1} \cdots i_{q}}{l_{\sigma}},
$$

where the tensor $\tilde{T}$ satisfies

$$
\tilde{T}_{i_{1} \cdots i_{k_{\sigma}} \cdots i_{l_{\sigma}} \cdots i_{q}} \cdot g^{i_{k_{\sigma}} i_{l_{\sigma}}}=0
$$

for all indices (2.1). The tensor $\tilde{T}$ is uniquely determined and $\stackrel{\left(k_{\sigma}, l_{\sigma}\right)}{M}$ are certain tensors of the type $(0, q-2)$.

Proof. Let $M_{n}$ be a Riemannian manifold with metric $g$. Now, let us consider an a priori chosen point $x_{0}$ at $M_{n}$. Values of metric $g$ as well as all the given tensors will be considered in $x_{0}$.

Taking a coordinate system in which the matrix of $g$ fulfills

$$
g_{i j}=g_{0} \cdot D, \text { where } g_{0} \in R \text {, and } D=\operatorname{diag}( \pm 1, \pm 1, \ldots, \pm 1),
$$

we obtain the inverse matrix $g^{-1}$ in the form of

$$
g^{i j}=g_{0}^{-1} \cdot D .
$$

The existence of the coordinated system above is guaranteed only for the concrete point $x_{0}$. A metric (2.4) exists globally in Euclidean and pseudo-Euclidean spaces.

Therefore, the relation (2.3) may be written as

$$
\sum_{k_{\sigma}, k_{\sigma}=1}^{n} \tilde{T}_{i_{1} \cdots i_{k \sigma} \cdots i_{l \sigma} \cdots i_{q}} \cdot g_{i_{k_{\sigma}} i_{l \sigma}}=0 .
$$

Let us define the inner product $\circ$ on $T_{q}^{0}$ by

$$
\stackrel{1}{T} \circ \stackrel{2}{T} \stackrel{\text { def }}{=} \sum_{i_{1}, \ldots, i_{q}=1}^{n} \stackrel{1}{T}_{i_{1} \cdots i_{q}} \cdot \stackrel{2}{T_{i_{1}} \cdots i_{q}} .
$$

Now, let us construct a linear subspace $T^{*} \subseteq \subseteq T_{p}^{0}$ which is generated by all the tensors of the form

$$
\sum_{\sigma=1}^{s} g_{i_{k_{\sigma}} i_{l \sigma}} \cdot \stackrel{\left(k_{\sigma}, l_{\sigma}\right)}{M} \underset{i_{1}, \cdots i_{k_{\sigma}-1} \curlyvee{ }_{i_{k_{\sigma}+1} \cdots i_{l_{\sigma}-1}}^{k_{\sigma}}{ }^{k_{\sigma}} i_{l_{\sigma}+1} \cdots i_{q}}{ } \cdot
$$


Considering a linear subspace in $T_{p}^{0}$ which contains all the tensors fulfilling (2.3), we obtain $\tilde{T} \circ \stackrel{*}{T}=0$ for any tensors $\tilde{T}$ of this subspace in the obvious way. Therefore, this subspace is contained in the orthogonal complement of the subspace $T^{*}$, which implies the unicity of the decomposition (2.2) of the tensor $T_{i_{1} \cdots i_{p}}$.

\section{SPECIAL DECOMPOSITIONS OF TENSORS}

Comparing the decomposition in the following theorem with decompositions in Theorem 1 (and Theorem 4) as well as in the fundamental theorem proved by Weyl (see [8]), we may remark that in the following one, not only tensor $\tilde{T}$ is uniquely determined but also all tensors $\stackrel{(*)}{M}$.

Theorem 2. Let $T_{i_{1} i_{2} \cdots i_{q}}$ be a $(0, q)$-tensor and

$$
\left(i_{1}, i_{2}\right),\left(i_{1}, i_{3}\right), \ldots,\left(i_{1}, i_{q}\right)
$$

be some pairs of indices. Then for $n>q-1$ there exists the unique decomposition of $T_{i_{1} \cdots i_{q}}$ of the form

$$
T_{i_{1} \cdots i_{q}}=\tilde{T}_{i_{1} \cdots i_{q}}+\sum_{\sigma=2}^{n} g_{i_{1} i_{\sigma}} \cdot \stackrel{(1, \sigma)}{M_{1}} \underset{i_{2}, \cdots i_{s-1} \curlyvee i_{s+1} \cdots i_{q}}{\sigma},
$$

where $\widetilde{T}_{i_{1} \cdots i_{q}}$ satisfies

$$
\tilde{T}_{i_{1} \cdots i_{\sigma} \cdots i_{q}} \cdot g^{i_{1} i_{\sigma}}=0
$$

for any pairs of indices (3.1). The tensor $\tilde{T}$ and all $(0, q-2)$-tensors $\stackrel{\left(i_{1} i_{\sigma}\right)}{M}$ are uniquely determined.

Proof. With respect to Theorem 1 we are only to prove the uniqueness of the tensors $\stackrel{\left(i_{1} i_{\sigma}\right)}{M}$. Contracting (3.2) with $g^{i_{1} i_{s}}$ for $s=2, \ldots, n$ and using (3.3) for the tensor $\tilde{T}$, we obtain the following system of linear equations

$$
\begin{aligned}
& T_{i_{1} \ldots i_{q}} g^{i_{1} i_{s}}=0+n \cdot \stackrel{(1, \sigma)}{M_{\curlyvee}} \underset{i_{2}, \cdots i_{s-1}}{\stackrel{\sigma}{\gamma} i_{s+1} \cdots i_{q}} \\
& +\sum_{\sigma=2, \sigma \neq s}^{q} M_{\curlyvee i_{2} \cdots i_{\sigma-1} \curlyvee i_{\sigma+1} \cdots i_{s-1} \stackrel{s}{\curlyvee} i_{s+1} \cdots i_{q}} .
\end{aligned}
$$

It follows from the fixed point theorem that such system of equations with variables $\stackrel{\left(i_{1} i_{2}\right)}{M}, \ldots, \stackrel{\left(i_{1} i_{n}\right)}{M}$ has for $n>q-1$ exactly one solution. 
Let us recall the raising indices of tensors. If given a tensor $T$ of the type $(0, p+q)$, we may construct a tensor of the type $(p, q)$ by the following:

$$
T_{j_{1} \cdots j_{q}}^{i_{1} \cdots i_{p}} \stackrel{\text { def }}{=} g^{i_{1} \alpha_{1}} g^{i_{2} \alpha_{2}} \cdots g^{i_{p} \alpha_{p}} T_{\alpha_{1} \cdots \alpha_{p} j_{1} \cdots j_{q}} .
$$

Raising indices, we obtain from Theorem 2 the known unique decomposition of the tensor $T_{i_{2} \cdots i_{p}}^{i_{1}}$ :

$$
T_{i_{2} \cdots i_{p}}^{i_{1}}=\widetilde{T}_{i_{2} \cdots i_{p}}^{i_{1}}+\delta_{i_{2}}^{i_{1}} M_{i_{3} \cdots i_{p}}^{12}+\delta_{i_{3}}^{i_{1}} M_{i_{2} i_{4} \cdots i_{p}}^{13}+\cdots+\delta_{i_{p}}^{i_{1}} M_{i_{2} \cdots i_{p-1}}^{1 p},
$$

where the tensor $\tilde{T}$ is traceless, i. e.,

$$
\tilde{T}_{\cdots \alpha \cdots}^{\alpha}=0
$$

and the tensor $\widetilde{T}$ as well as the $(0, p-1)$-tensors $\stackrel{1 *}{M}$ are uniquely determined.

As we have mentioned above, the decomposition (3.6) is presented by Weyl in [8], but the uniqueness of tensors $\stackrel{1 *}{M}$ is not contained there.

In view of (3.5), Theorem 1 implies immediately

Theorem 3. Let $T_{i_{1} \cdots i_{p}}^{j_{1} \cdots j_{q}}$ be a $(p, q)$-tensor and

$$
\left(i_{k_{1}}, j_{l_{1}}\right),\left(i_{k_{2}}, j_{l_{2}}\right), \ldots,\left(i_{k_{s}}, j_{l_{s}}\right), s \leq p q,
$$

be pairs of indices satisfying $1 \leq k_{\sigma} \leq p, 1 \leq l_{\sigma} \leq q$.

Then there exists a decomposition of $T_{i_{1} \cdots i_{p}}$ of the form

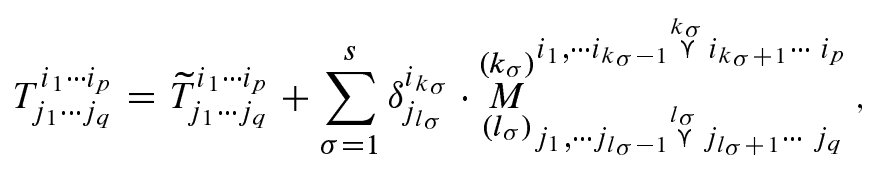

where the tensor $\tilde{T}$ satisfies

$$
\widetilde{T}_{j_{1} \cdots j_{l_{\sigma}} \cdots j_{q}}^{i_{1} \cdots i_{k_{\sigma}} \cdots i_{p}} \cdot \delta_{i_{k_{\sigma}}}^{j_{l_{\sigma}}}=0
$$

for any indices (3.8). The tensor $\tilde{T}$ is uniquely determined and $\underset{\left(l_{\sigma}\right)}{\stackrel{\left(k_{\sigma}\right)}{M}}$ are certain tensors of the type $(p-1, q-1)$.

The condition (3.10) means that the tensor $\tilde{T}$ is traceless over any pair of indices (3.8). This may be expressed by

$$
\tilde{T}_{j_{1} \cdots j_{l_{\sigma-1}} \alpha j_{l_{\sigma}+1} \cdots j_{q}}^{i_{1} \cdots i_{k_{\sigma}} \alpha i_{k_{\sigma}} \cdots i_{p}}=0 .
$$

Let us remark that Theorem 3 holds on every manifold since the metric tensor (which was used in the proof of Theorem 1) may be constructed in any case.

Immediately, we obtain the following Theorem, which is presented in $[3,6]$. 
Theorem 4. Let $T_{i_{1} \cdots i_{p}}^{j_{1} \cdots j_{p}}$ be a tensor of the type $(p, q)$. If $n+1 \geq p+q$, then there exists the unique decomposition of $T_{i_{1} \cdots i_{p}}^{j_{1} \cdots j_{p}}$ in the following form.

$$
T_{i_{1} \cdots i_{p}}=\tilde{T}_{i_{1} \cdots i_{p}}+\sum_{t=1}^{\min \{p, q\}} \sum_{\oplus} \delta_{j_{\sigma_{1}}}^{i_{\rho_{1}}} \delta_{j_{\sigma_{2}}}^{i_{\rho_{2}}} \cdots \delta_{j_{\sigma_{t}}}^{i_{\rho_{t}}} \stackrel{\star}{M} \cdots
$$

where

$$
\begin{aligned}
& \oplus= \begin{cases}\rho_{1}, \rho_{2}, \ldots, \rho_{t}=1,2, \ldots, p & \left(\rho_{1}<\rho_{2}<\cdots<\rho_{t}\right) \\
\sigma_{1}, \sigma_{2}, \ldots, \sigma_{t}=1,2, \ldots, q & \left(\sigma_{i} \text { are mutually different }\right),\end{cases} \\
& \star=\left\{\begin{array}{llll}
\rho_{1} & \rho_{2} & \cdots & \rho_{t} \\
\sigma_{1} & \sigma_{2} & \cdots & \sigma_{t}
\end{array}\right\}
\end{aligned}
$$

and tensors $\widetilde{T}_{i_{1} \cdots i_{p}}$ and $\stackrel{*}{M}$ are traceless.

\section{SPECIAL DIFFERENTIAL OPERATOR $L$}

Now, let us introduce the following concept on tensors of the type $(p, q)$.

Definition 1. A map $L: T_{q}^{p} \mapsto T_{q}^{p}$ is said to be a special differential operator if for any tensors $\stackrel{1}{T}, \stackrel{2}{T}$ the following properties are satisfied:

$$
\begin{gathered}
L(\stackrel{1}{T} \pm \stackrel{2}{T})=L \stackrel{1}{T} \pm \stackrel{2}{T}, \\
L(\stackrel{1}{T} \cdot \stackrel{2}{T})=L \stackrel{1}{T} \cdot \stackrel{2}{T}+\stackrel{1}{T} \cdot L \stackrel{2}{T}, \\
L g=0 ; \quad L \&=0 .
\end{gathered}
$$

By $\delta$ we denote the Kronecker tensor.

Let us give some examples of the operator $L$ :

(a) $\nabla$ - the covariant derivative, i. e., $\nabla$ is an affine connection on $M_{n}$

(b) $\circ R$ - "semisymmetric" operator; $T \circ R$ is in the coordinate description defined by

$$
\nabla_{l} \nabla_{m} T_{i_{1} \ldots i_{q}}^{h_{1} \ldots h_{p}}-\nabla_{m} \nabla_{l} T_{i_{1} \ldots i_{q}}^{h_{1} \ldots h_{p}} .
$$

Using the Bianci identity, we may write $T \circ R$ in the form

$$
-T_{i_{1} \ldots i_{q}}^{\alpha \ldots h_{p}} R_{\alpha l m}^{h_{1}}-\cdots-T_{i_{1} \ldots i_{q}}^{h_{1} \ldots \alpha} R_{\alpha l m}^{h_{p}}+T_{\alpha \ldots i_{q}}^{h_{1} \ldots h_{p}} R_{i_{1} l m}^{\alpha}+\cdots+T_{i_{1} \ldots \alpha}^{h_{1} \ldots h_{p}} R_{i_{q} l m}^{\alpha},
$$

where $R_{i j k}^{h}$ are components of a Riemannian tensor $R$ on $M_{n}$. 
(c) $\circ Z$ - "pseudosymmetric" operator; this operator is defined analogously as the previous one - in the formula (4.2), we replace the tensor $R$ by the tensor $Z$ defined as follows

$$
Z_{i j k}^{h}=R_{i j k}^{h}-B\left(\delta_{k}^{h} g_{i j}-\delta_{j}^{h} g_{i k}\right),
$$

where $B$ is a function on $M_{n}$.

\section{ON MANIFOLDS WITH $L T=0$}

It is well known that a symmetric manifold in the sense of Cartan is characterized by the condition

$$
\nabla R=0 .
$$

Projective symmetric and conformal symmetric manifolds are characterized by the conditions

$$
\nabla W=0 \text { and } \nabla C=0 \text {, respectively; }
$$

by $W$ and $C$ we denote the Weyl tensors of projective and conformal curvature, respectively.

Semisymmetric and pseudosymmetric manifolds are characterized by the conditions

$$
R \circ R=0 \text { and } R \circ Z=0 \text {, respectively. }
$$

Further, projective and conformal semisymmetric manifolds are characterized by

$$
W \circ R=0 \text { and } C \circ R=0 \text {, respectively. }
$$

Replacing in (5.1) the Riemannian tensor by the Weyl tensor, we obtain the characterization of projective pseudosymmetric and conformal pseudosymmetric manifolds.

It follows from these remarks that it is useful to investigate manifolds with a general condition $L T=0$.

Applying Definition 1 of the operator $L$ and Theorem 1, we may prove the following lemmas.

Lemma 1. Let $T$ be a tensor of the type $(0, p)$ with the decomposition (2.2) for the selected pairs of indices (2.1). If $L \tilde{T}=0$ and $L \stackrel{*}{M}=0$, where $*=\left(k_{\sigma}, l_{\sigma}\right)$, then $L T=0$.

Lemma 2. Let $T$ be a tensor of the type $(0, p)$ with the decomposition (2.2) for the selected pairs of indices (2.1). If $L T=0$ then $L \widetilde{T}=0$.

Consider a $C^{\infty}(M)$-module of $k$-differential forms $\Omega^{k}(M)$ on a manifold $M_{n}$. Then for any $k$-differential form $\alpha \in \Omega^{k}(M)$, the special differential operator $L$ may be determined by the relation $L T=\alpha T$.

Let a 1-differential form $\omega \in \Omega^{1}(M)$ with

$$
\nabla_{X} T=\omega(X) \cdot T
$$


be given (see [1]). Then we speak about T-recurrency. The following special cases are well-known:

$\diamond \nabla R=\omega \cdot R$, the Walker recurrency (or simply recurrency),

$\diamond \nabla W=\omega \cdot W$, the projective recurrency,

$\diamond \nabla C=\omega \cdot C$, the conformal recurrency,

$\diamond \nabla$ Ric $=\omega \cdot$ Ric, the Ricci recurrency.

Therefore, it will be useful to deal with the case (5.2).

Considering a 1-differential form $\omega$ with (5.2) and choosing indices according to the Theorem 2, we may prove the following theorem.

Theorem 5. Let $T$ be a $(0, p)$-tensor decomposed in the sense of (3.2). Then

$$
L T=\omega T,
$$

if and only if

$$
L \tilde{T}=\omega \tilde{T} \quad \text { and } \quad L \stackrel{1 \sigma}{M}=\omega \stackrel{1 \sigma}{\omega}
$$

where $\omega$ is a $k$-differential form in $\Omega^{k}(M)$.

Proof. Consider a decomposition (3.2) of $T$ for pairs of indices (3.1). First, let us suppose

$$
L \tilde{T}=\omega \tilde{T} \quad \text { and } \quad \stackrel{1 \sigma}{M}=\omega \stackrel{1 \sigma}{\omega} .
$$

Substituting (5.5) into (3.2), we obtain immediately $L T=\omega T$.

Now, let us suppose $L T=\omega T$. Applying $L$ to the expression (3.2) of $T$, we have

$$
\begin{aligned}
L T_{i_{1} \cdots i_{p}}=L \widetilde{T}_{i_{1} \cdots i_{p}}+g_{i_{1} i_{2}} L \stackrel{12}{M}_{i_{3} \cdots i_{p}}+g_{i_{1} i_{3}} L \stackrel{13}{M}_{i_{2} i_{4} \cdots i_{p}} & +\cdots \\
& +g_{i_{1} i_{p}} L \stackrel{1 p}{M}_{i_{2} \cdots i_{p-1}}
\end{aligned}
$$

i. e.

$$
\begin{aligned}
\omega T=L \widetilde{T}_{i_{1} \cdots i_{p}}+g_{i_{1} i_{2}} L \stackrel{12}{M}_{i_{3} \cdots i_{p}}+g_{i_{1} i_{3}} L \stackrel{13}{M}_{i_{2} i_{4} \cdots i_{p}} & \\
& \\
& +\cdots+g_{i_{1} i_{p}} L \stackrel{1 p}{M}_{i_{2} \cdots i_{p-1}} .
\end{aligned}
$$

Applying the form $\omega$ to the expression of $T$ in the form (3.2), we obtain

$$
\begin{aligned}
\omega T=L \widetilde{T}_{i_{1} \cdots i_{p}}+g_{i_{1} i_{2}} \omega \stackrel{12}{M}_{i_{3} \cdots i_{p}}+g_{i_{1} i_{3}} \omega \stackrel{13}{M}_{i_{2} i_{4} \cdots i_{p}} & \\
& +\cdots+g_{i_{1} i_{p}} \omega M_{i_{2} \cdots i_{p-1}} .
\end{aligned}
$$


Comparing (5.7) and (5.8), we have

$$
\begin{aligned}
& (L \widetilde{T}-\omega \widetilde{T})+\left(g_{i_{1} i_{2}} L \stackrel{12}{M}_{i_{3} \cdots i_{p}}-g_{i_{1} i_{2}} \omega \stackrel{12}{M}_{i_{3} \cdots i_{p}}\right) \\
& +\left(g_{i_{1} i_{3}} L \stackrel{13}{M} i_{i_{2} i_{4} \cdots i_{p}}-g_{i_{1} i_{3}} \omega \stackrel{13}{M}_{i_{2} i_{4} \cdots i_{p}}\right)+ \\
& +\cdots+\left(g_{i_{1} i_{p}} L{\stackrel{1 p}{M_{i_{2} \cdots i_{p-1}}}}-g_{i_{1} i_{p}} \omega M_{i_{2} \cdots i_{p-1}}\right)=0 .
\end{aligned}
$$

Contracting (5.9) with $g^{i_{1} i_{\sigma}}$ for $\sigma=2, \ldots, n$, we deduce a system of linear equations as follows

$$
n \cdot(L \stackrel{1 \sigma}{M}-\omega \stackrel{1 \sigma}{M})+\sum_{\tau=2, \tau \neq \sigma}^{p}(L \stackrel{1 \tau}{M}-\omega \stackrel{1 \tau}{M})=0 .
$$

For $n>p-1$, such a system has the unique solution $L \stackrel{1 \sigma}{M}-\omega \stackrel{1 \sigma}{M}=0$ for $\sigma=$ $2, \ldots, n$, which implies that $L \widetilde{T}-\omega T=0$.

The following theorem is the consequence of Theorem 5 .

Theorem 6. Let $T$ be a $(0, p)$-tensor decomposed in the sense of (3.2). Then

$$
L T=0
$$

if and only if $L \tilde{T}=0$ and $L \stackrel{1 \sigma}{M}=0$.

\section{REFERENCES}

[1] M. Crasmareanu, "Trace Decomposition and Recurrency.” Acta Univ. Palacki. Olomuc., Fac. rer. nat., Mathematica, vol. 40, pp. 43-46, 2001.

[2] M. Jukl, L. Juklová, and J. Mikeš, "The decomposition of tensor spaces with quaternionic structure," in APLIMAT 2007, Bratislava, 2007, pp. 217-222.

[3] D. Krupka, "The trace decomposition of tensor spaces," Linear and Multilinear Algebra, vol. 54, no. 4, pp. 235-263, 2006.

[4] L. Lakomá and M. Jukl, "The decomposition of tensor spaces with almost complex structure," Rendiconti del circolo matematico di Palermo, Ser. II, Suppl., vol. 72, pp. 145-150, 2004.

[5] L. Lakomá and J. Mikeš, "On the special trace decomposition problem on quaternionic structure," in Proc. of the Third Internat. Workshop on Diff. Geom. and its Appl.; The First German-Romanian Seminar on Geom., Sibiu, Romania, 1997, pp. 225-229.

[6] J. Mikeš, "On general trace decomposition problem," in Diff. Geom. and its Appl., Proc. of 6th Int. Conf., Brno, Czech Republic, 1995, pp. 45-50.

[7] V. V. Vinshnevskij, A. P. Shirokov, and V. V. Shurygin, Spaces over algebras. (Prostranstva nad algebrami). Kazan: Izd. Kazan. Univ., 1985.

[8] H. Weyl, The classical groups. Princeton: Princeton Univ. Press,, 1946, fifteenth printing (1997).

[9] K. Yano, Differential geometry on complex and almost complex spaces. Pergamon press, 1965. 
Authors' addresses

\section{Marek Jukl}

Palacký University, Department of Algebra and Geometry, Faculty of Science, Tř. 17. listopadu 12, 77146 Olomouc, Czech Republic

E-mail address: marek. jukl@upol.cz

Lenka Juklová

Palacký University, Department of Algebra and Geometry, Faculty of Science, Tř. 17. listopadu 12, 77146 Olomouc, Czech Republic

E-mail address: lenka.juklova@upol.cz 